\title{
Populações vulneráveis e o desfecho dos casos de tuberculose no Brasil
}

\author{
Vulnerable populations and tuberculosis treatment outcomes \\ in Brazil
}

Laylla Ribeiro Macedo (http://orcid.org/0000-0002-6246-3559) ${ }^{1}$

Ethel Leonor Noia Maciel (http://orcid.org/0000-0003-4826-3355) ${ }^{1}$

Claudio Jose Struchiner (http://orcid.org/0000-0003-2114-847X) ${ }^{2}$

${ }^{1}$ Universidade Federal do Espírito Santo. Av. Fernando Ferrari 514, Goiabeiras. 29075-910 Vitória ES Brasil.

layllarm@hotmail.com

${ }^{2}$ Universidade Estadual do Rio de Janeiro. Rio de Janeiro RJ Brasil.

\begin{abstract}
This article aims to assess the association between being a prisoner or homeless and treatment failure in cases of tuberculosis diagnosed in Brazil in 2015. We examined cases of tuberculosis in prisoners and the homeless in Brazil in 2015 reported to the national notifiable diseases information system using descriptive analysis and logistic regression. There were 82,056 cases of tuberculosis in 2015. Of these, 7,462 (10.3\%) were prisoners and 2,782 (3.9\%) were homeless. The rate of treatment success in prisoners was $78.6 \%$, while the rate of failure in the homeless was $63.2 \%$. Being a prisoner was a protective factor against treatment failure (adjusted odds ratio 0.68, 95\% CI 0.63-0.73), while being homeless was a risk factor for treatment failure (adjusted odds ratio 2.38 , 95\%CI 2.17-2.61). Treatment success and failure rates differed between prisoners and the homeless. Our findings reinforce the need for public health policies tailored to the specific needs of these groups implemented in conjunction with social services and public security agencies in order to have a significant impact on TB incidence. Key words Tuberculosis, Vulnerable populations, Prisons, The Homeless
\end{abstract}

Resumo $O$ objetivo deste artigo é avaliar a associação entre estar privado de liberdade ou em situação de rua e o insucesso no desfecho dos casos de tuberculose diagnosticados no Brasil em 2015. Foram estudados casos de tuberculose na população privada de liberdade e em situação de rua do Brasil em 2015, por análise descritiva e regressão logística baseado no Sistema de Informação de Agravos de Notificação. Em 2015 notificou-se 82.056 casos de tuberculose, destes 7.462 (10,3\%) estavam privados de liberdade e $2.782(3,9 \%) \mathrm{em}$ situação de rua. A proporção de sucesso no desfecho dos casos na população privada de liberdade foi de 78,6\%, enquanto na população em situação de rua, a proporção de insucesso foi 63,2\%. Estar privado de liberdade mostrou-se protetor para o insucesso no desfecho dos casos (Razão de Chances ajustada 0,68, IC95\% 0,63-0,73), enquanto estar em situação de rua mostrou-se um fator de risco para o insucesso (Razão de Chances ajustada 2,38, IC95\% 2,17-2,61). O desfecho dos casos de tuberculose se diferencia entre as populações privada de liberdade e em situação de rua, fazendo-se necessário a implantação de políticas públicas de saúde que considerem suas especificidades e seja articulada com órgãos sociais e segurança a fim de que impacte nos indicadores da doença.

Palavras-chave Tuberculose, Populações vulneráveis, Prisões, Pessoas em situação de rua 


\section{Introdução}

A tuberculose (TB) é principal causa de morte entre as doenças infecciosas em todo o mundo ${ }^{1}$. Sabe-se que a pobreza é uma das causas da TB, e que o adoecimento gera ainda mais pobreza, mantendo ativo um ciclo que envolve indivíduos, famílias e comunidades ${ }^{1}$.

Estudos apontam a existência de uma associação direta entre os indicadores socioeconômicos e a ocorrência da doença, tanto em nível individual quanto coletivo, enfatizando que a TB está relacionada intimamente com as condições de vida dos indivíduos e seu meio social ${ }^{2}$.

Nesse contexto, alguns grupos populacionais estão mais vulneráveis e destacam-se por apresentarem índices da doença ainda mais elevados ${ }^{3}$. Dados mostram que o risco de adoecimento por TB na população privada de liberdade (PPL) e na população em situação de rua (PSR) é 28 e 56 vezes maior respectivamente, quando comparados com o risco de adoecimento da população em geral.

Alguns fatores são conhecidos pela literatura como potenciais influenciadores no desfecho do tratamento da tuberculose, sendo eles: sexo, idade, escolaridade, forma da TB, agravos associados principalmente Aids, diabetes e doenças mentais, uso de álcool, tabaco ou drogas, dentre outros ${ }^{4}$. Porém há uma carência de informações sobre como as populações privadas de liberdade ou em situação de rua no Brasil podem se relacionar com o desfecho da doença.

Frente à complexidade dos determinantes sociais da TB, o pilar 2 da Estratégia de Controle da Tuberculose pós 2015 recomenda políticas de proteção social para os pacientes e acesso universal à saúde ${ }^{5}$, tornando essencial conhecer o comportamento da doença nesses grupos vulneráveis.

Diante disso, o objetivo deste estudo é avaliar a associação entre estar privado de liberdade ou em situação de rua e o insucesso no desfecho dos casos de tuberculose diagnosticados no Brasil no ano de 2015.

\section{Métodos}

A população do estudo foram os casos diagnosticados com tuberculose no Brasil no ano de 2015, notificados no Sistema de Informação de Agravos de Notificação (Sinan). Foram excluídos indivíduos abaixo de 15 anos, devido ao comportamento diferenciado da doença nessa faixa etária.

Neste estudo, as populações vulneráveis foram definidas como os casos que estavam priva- dos de liberdade ou em situação de rua, categorizados a partir da variável "situações especiais" preenchida na Ficha de Notificação/Investigação de Tuberculose do Sinan no momento de notificação da doença. Essa variável foi incluída na ficha a partir da sua última revisão no final do ano de 2014.

Para o desfecho dos casos foram consideradas inicialmente as categorias da Ficha de Acompanhamento de Tuberculose do Sinan, sendo elas: cura, abandono (perda de seguimento), óbito por tuberculose, óbito por outras causas, transferência, tuberculose multidroga resistente (TB-MDR), mudança de esquema, falência e abandono primário (perda de seguimento primário). Para a regressão, essas categorias foram reagrupadas em sucesso (cura) e insucesso no desfecho dos casos (todas as demais).

Outras variáveis foram incluídas no estudo por se tratar de possíveis fatores de confusão com base na literatura. Essas variáveis foram: sexo, idade, raça/cor, escolaridade, região de residência, tipo de entrada, forma da tuberculose, doenças e agravos associados (Aids, diabetes mellitus, doença mental, alcoolismo, tabagismo e drogas), exames (raio x, baciloscopia de escarro e cultura inicial) e tratamento supervisionado realizado. Ressalta-se que no caso da variável escolaridade, a categoria "não se aplica" é utilizada para pacientes com idade inferior a 7 anos.

Os dados utilizados no estudo referem-se ao banco do Sinan, fornecido pelo Ministério da Saúde (MS) e atualizado em fevereiro de 2017. O Sinan é um sistema nacional de informações que visa apoiar a coleta e o processamento de dados sobre agravos, doenças e eventos de saúde pública, incluindo a TB. As informações geradas auxiliam no planejamento, prevenção, avaliação e controle de doenças, atuando como uma importante ferramenta para vigilância epidemiológica ${ }^{6}$.

Os casos foram apresentados de acordo com suas características sociodemográficas e clínicas, agrupados por tipo de população: população total (todos os casos notificados), população privada de liberdade e não privada de liberdade, e população em situação de rua e não em situação de rua. Para cada variável foi calculado o teste quiquadrado ou exato de Fisher a fim de comparar as proporções entre as populações e seu controle.

No estudo foram utilizados dois modelos de regressão logística para avaliar a associação entre estar privado de liberdade ou em situação de rua e o insucesso no desfecho dos casos de tuberculose, separadamente. Para isso, foi realizada uma imputação múltipla dos dados faltantes, seguindo as etapas sugeridas por Harrell ${ }^{7}$. Inicialmente 
foi investigado o padrão dos dados sem registro, observando que os dados faltantes de uma dada variável estavam relacionados aos dados preenchidos de outra variável do modelo - "missing at random" (MAR). O método utilizado foi o "predictive mean matching" (PMM), que propõe substituir o dado faltante de uma variável a partir de uma observação doadora (média predita) ${ }^{7}$. Foram consideradas na imputação as seguintes variáveis: raça/cor, escolaridade, tipo de entrada, forma da TB, agravos associados como Aids, diabetes mellitus, doença mental, álcool, tabaco e drogas, exames de raio $\mathrm{x}$, baciloscopia e cultura de escarro, tratamento supervisionado realizado e as variáveis população privada de liberdade e população em situação de rua. Para a imputação gerou-se dez conjuntos de dados, utilizando como variáveis auxiliares aquelas incluídas nos modelos finais.

Para obter uma estimativa da associação entre estar privado de liberdade ou em situação de rua e o insucesso no desfecho dos casos de TB foram ajustadas as regressões logísticas incluindo todos os confundidores potenciais a priori selecionados. Para os modelos finais de regressão logística a partir dos dados imputados, testou-se a hipótese utilizando o teste Wald e para os intervalos de confiança de 95\% (IC95\%), os intervalos de Wald.

Para as análises estatísticas foi utilizado o programa $\mathrm{R}$ na versão 3.4.1 e para imputação foram utilizados os pacotes Hmisc e Rms.

Essa pesquisa foi aprovada pelo Comitê de Ética em Pesquisa (CEP) da Escola Nacional de Saúde Pública Sérgio Arouca (ENSP) da Fundação Oswaldo Cruz (Fiocruz).

\section{Resultados}

No ano de 2015 foram notificados 84.405 casos confirmados de tuberculose no Brasil. Após a exclusão de $2.349(2,8 \%)$ indivíduos com idade abaixo de 15 anos, totalizou-se 82.056 casos estudados. Destes $7.462(10,3 \%)$ se encontraram privados de liberdade e $2.782(3,9 \%)$ em situação de rua.

Os casos diagnosticados com tuberculose nas populações privada de liberdade e em situação de rua apresentaram características sociodemográficas semelhantes mostrando-se respectivamente em sua maioria homens (95,6\% e 79,9\%), jovens de 15 a 39 anos $(85,3 \%$ e $53,1 \%)$, pardos $(44,1 \%$ e $44,1 \%)$, com 5 a 8 anos de estudo $(34,7 \%$ e $27,6 \%)$ e residentes da região sudeste $(53,6 \%$ e $54,5 \%$ ) (Tabela 1).
Comparando as populações (população privada de liberdade vs. população em situação de rua) quanto às características clínicas, destacase na PPL mais casos novos $(75,2 \%$ vs. $56,0 \%)$ e menos reingressos após perda de seguimento (11,9\% vs. $33,7 \%)$; menor prevalência de agravos associados como Aids ( $7,6 \%$ vs. $23,8 \%$ ), diabetes $(1,4 \%$ vs. $3,1 \%)$, doença mental ( $1,2 \%$ vs. $6,7 \%)$, alcoolismo (10,5\% vs. $52,5 \%)$, tabagismo $(21,0 \%$ vs. $42,2 \%)$ e uso de drogas $(19,2 \%$ vs. $53,0 \%)$; além de mais realização de tratamento supervisionado (42,0\% vs. $33,2 \%)$. Quanto ao desfecho dos casos na população em situação de rua observou-se menor frequência de cura $(34,7 \%$ vs. $67,9 \%)$, maior de perda de seguimento $(33,7 \%$ vs. 7,6\%); e óbito por TB (5,9\% vs. $1,0 \%)$ e outras causas $(5,7 \%$ vs. $1,6 \%)$ (Tabela 2$)$.

Embora o desfecho dos casos na população total e PPL se apresentem de forma semelhante, se observa que a proporção de sucesso na PPL $(78,6 \%)$ ainda é superior à da população total (70,4\%). Na população em situação de rua notase o inverso do observado nas demais populações (população total e PPL), mostrando uma proporção de insucesso $(63,2 \%)$ superior à do sucesso $(36,8 \%)$ no desfecho dos casos (Gráfico 1).

Vale ressaltar que neste estudo foram incluídos os casos de tuberculose para todos os tipos de entrada, porém em uma análise comparativa entre os grupos de casos com todos os tipos de entrada e aquele com somente casos novos, notouse que embora para os casos novos o percentual de cura (desfecho de sucesso) tenha se mostrado um pouco superior, isso ocorreu de forma proporcional para todas as populações (população total, população privada de liberdade e população em situação de rua).

A Tabela 3 apresenta os modelos finais para a população privada de liberdade e população em situação de rua separadamente. Observou-se que estar privado de liberdade mostrou-se um fator protetor do insucesso no desfecho dos casos (RCajustada 0,68, IC95\% 0,63-0,73), enquanto estar em situação de rua mostrou ser um fator de risco ao insucesso (RCajustada 2,38, IC95\% 2,172,61), mesmo após os ajustes.

\section{Discussão}

No ano de 2015, a população privada de liberdade e a população em situação de rua representaram $10,3 \%$ e $3,9 \%$ do total de casos notificados no período respectivamente. As características sociodemográficas das populações privada de liberdade e em situação de rua observadas no 
Tabela 1. Características sociodemográficas dos casos diagnosticados com tuberculose segundo o tipo de população. Brasil, 2015.

\begin{tabular}{|c|c|c|c|c|c|}
\hline $\begin{array}{l}\text { Características } \\
\text { sociodemográficas }\end{array}$ & $\begin{array}{c}\text { População total } \\
\mathrm{n}=82.056(\%)\end{array}$ & $\begin{array}{c}\text { População } \\
\text { privada de } \\
\text { liberdade } \\
\mathrm{n}=7.462(\%)\end{array}$ & $\begin{array}{c}\text { População não } \\
\text { privada de } \\
\text { liberdade } \\
\mathbf{n}=65.230(\%)\end{array}$ & $\begin{array}{c}\text { População em } \\
\text { situação de rua } \\
\text { n=2.782 (\%) }\end{array}$ & $\begin{array}{c}\text { População não } \\
\text { em situação de } \\
\text { rua } \\
n=69.318(\%)\end{array}$ \\
\hline \multicolumn{6}{|l|}{ Sexo } \\
\hline Masculino & $56.972(69,4)$ & $7.133(95,6)$ & $43.498(66,7)$ & $2.223(79,9)$ & $47.930(69,1)$ \\
\hline Feminino & $25.084(30,6)$ & $329(4,4)$ & $21.732(33,3)$ & $559(20,1)$ & $21.388(30,9)$ \\
\hline \multicolumn{6}{|l|}{ Faixa etária } \\
\hline 15 a 39 anos & $43.013(52,5)$ & $6.364(85,3)$ & $32.170(49,3)$ & $1.477(53,1)$ & $36.636(52,8)$ \\
\hline 40 a 64 anos & $31.385(38,2)$ & $1.008(13,5)$ & $26.539(40,7)$ & $1.229(44,2)$ & $26.181(37,8)$ \\
\hline 65 ou mais anos & $7.658(9,3)$ & $90(1,2)$ & $6.521(10,0)$ & $76(2,7)$ & $6.501(9,4)$ \\
\hline \multicolumn{6}{|l|}{ Raça/Cor } \\
\hline Branca & $25.726(31,4)$ & $2.360(31,6)$ & $21.407(32,8)$ & $739(26,6)$ & $22.900(33,0)$ \\
\hline Preta & $10.548(12,9)$ & $881(11,8)$ & $8.400(12,9)$ & $567(20,4)$ & $8.649(12,5)$ \\
\hline Amarela & $570(0,7)$ & $57(0,8)$ & $418(0,6)$ & $12(0,4)$ & $458(0,7)$ \\
\hline Parda & $37.828(46,0)$ & $3.290(44,1)$ & $29.687(45,6)$ & $1.228(44,1)$ & $31.429(45,3)$ \\
\hline Indígena & $785(1,0)$ & $31(0,4)$ & $662(1,0)$ & $7(0,3)$ & $680(1,0)$ \\
\hline Missing & $6.599(8,0)$ & $843(11,3)$ & $4.656(7,1)$ & $229(8,2)$ & $5.202(7,5)$ \\
\hline \multicolumn{6}{|l|}{ Escolaridade } \\
\hline Analfabeto & $3.196(3,9)$ & $162(2,2)$ & $2.553(3,9)$ & $119(4,3)$ & $2.573(3,7)$ \\
\hline 1 a 4 anos & $14.484(17,7)$ & $1.154(15,5)$ & $11.531(17,7)$ & $575(20,7)$ & $11.987(17,3)$ \\
\hline 5 a 8 anos & $20.436(24,9)$ & $2.593(34,7)$ & $16.070(24,6)$ & $770(27,6)$ & $17.732(25,6)$ \\
\hline$>8$ anos & $21.487(26,2)$ & $1.389(18,6)$ & $18.433(28,3)$ & $328(11,8)$ & $19.407(28,0)$ \\
\hline Não se aplica & $434(0,5)$ & $32(0,4)$ & $402(0,6)$ & $26(0,9)$ & $408(0,6)$ \\
\hline Missing & $22.019(26,8)$ & $2.132(28,6)$ & $16.241(24,9)$ & $964(34,7)$ & $17.211(24,8)$ \\
\hline \multicolumn{6}{|l|}{ Região } \\
\hline Norte & $8.342(10,2)$ & $477(6,4)$ & $6.891(10,6)$ & $134(4,8)$ & $7.181(10,4)$ \\
\hline Nordeste & $21.512(26,2)$ & $1.458(19,5)$ & $15.779(24,2)$ & $444(16,0)$ & $16.433(23,7)$ \\
\hline Sudeste & $37.732(46,0)$ & $3.977(53,3)$ & $30.479(46,7)$ & $1.517(54,5)$ & $32.817(47,3)$ \\
\hline Sul & $10.697(13,0)$ & $1.116(15,0)$ & $9.292(14,2)$ & $568(20,4)$ & $9.802(14,1)$ \\
\hline Centro-Oeste & $3.773(4,6)$ & $434(5,8)$ & $2.789(4,3)$ & $119(4,3)$ & $3.085(4,5)$ \\
\hline
\end{tabular}

Fonte: Elaborado pelos autores.

estudo, se aproximam do perfil dos casos diagnosticados nessas populações já descritos ${ }^{8,9}$, reafirmando a associação entre o adoecimento por tuberculose e as questões relativas ao indivíduo e condições socioambientais ${ }^{2,10}$.

Vale destacar, na população em situação de rua, a proporção de reingresso após perda de seguimento acima de $30 \%$, enquanto na população que não estava em situação de rua esse quantitativo foi inferior (8,5\%). Questões como uso diário de álcool, uso de drogas, injetáveis ou não, e desemprego contribuem significativamente para essa questão ${ }^{11}$. A prevalência dos agravos associados na população em situação de rua também se mostrou superior a encontrada nas demais populações, realçando uma característica comum a esse grupo ${ }^{12,13}$ que interfere diretamente no trata- mento da TB. Na população privada de liberdade esse quantitativo foi semelhante ao encontrado na população total.

Na população privada de liberdade, destacase a proporção de tratamento diretamente observado (TDO) $(42,6 \%)$ maior do que a registrada na não PPL (34,2\%), semelhante ao observado em um estudo norte americano onde $65,0 \%$ da PPL recebia TDO vs. $41,0 \%$ da não $\mathrm{PPL}^{14}$. Ainda que a PPL tenha apresentado uma proporção de tratamento supervisionado para TB superior aos demais grupos, esse quantitativo ainda é bastante inferior ao $100 \%$ preconizado pelo $\mathrm{MS}^{15}$.

Embora as populações privadas de liberdade e em situação de rua sejam consideradas populações vulneráveis para a tuberculose e apresentem uma incidência da doença superior à observada 
Tabela 2. Características clínicas dos casos diagnosticados com tuberculose segundo o tipo de população. Brasil, 2015.

\begin{tabular}{|c|c|c|c|c|c|}
\hline Características clínicas & $\begin{array}{c}\text { População } \\
\text { total } \\
\mathrm{n}=82.056(\%)\end{array}$ & $\begin{array}{c}\text { População } \\
\text { privada de } \\
\text { liberdade } \\
\mathbf{n}=7.462(\%)\end{array}$ & $\begin{array}{c}\text { População } \\
\text { não privada } \\
\text { de liberdade } \\
\mathrm{n}=65.230(\%)\end{array}$ & $\begin{array}{c}\text { População } \\
\text { em situação } \\
\text { de rua } \\
\text { n=2.782 }(\%)\end{array}$ & $\begin{array}{l}\text { População não } \\
\text { em situação } \\
\text { de rua } \\
\text { n=69.318 (\%) }\end{array}$ \\
\hline \multicolumn{6}{|l|}{ Entrada } \\
\hline Caso novo & $65.998(80,4)$ & $4.614(75,2)$ & $53.083(81,3)$ & $1.559(56,0)$ & $56.662(81,7)$ \\
\hline Recidiva & $5.532(6,7)$ & $763(10,2)$ & $4.170(6,4)$ & $196(7,0)$ & $4.693(6,8)$ \\
\hline $\begin{array}{l}\text { Reingresso após perda de } \\
\text { seguimento }\end{array}$ & $7.757(9,5)$ & $891(11,9)$ & $5.989(9,2)$ & $935(33,7)$ & $5.892(8,5)$ \\
\hline Não sabe & $248(0,3)$ & $12(0,2)$ & $139(0,2)$ & $19(0,7)$ & $131(0,2)$ \\
\hline Transferência & $2.197(2,7)$ & $176(2,4)$ & $1.682(2,6)$ & $65(2,3)$ & $1.774(2,6)$ \\
\hline Pós-óbito & $324(0,4)$ & $6(0,1)$ & $167(0,3)$ & $8(0,3)$ & $166(0,2)$ \\
\hline \multicolumn{6}{|l|}{ Forma } \\
\hline Pulmonar & $69.765(85,0)$ & $7.038(94,3)$ & $54.487(83,5)$ & $2.545(91,5)$ & $58.450(84,3)$ \\
\hline Extrapulmonar & $9.746(11,9)$ & $310(4,2)$ & $8.578(13,2)$ & $125(4,5)$ & $8.720(12,6)$ \\
\hline Pulmonar+Extrapulmonar & $2.504(3,1)$ & $114(1,5)$ & $2.160(3,3)$ & $112(4,0)$ & $2.143(3,1)$ \\
\hline Missing & $41(0)$ & $0(0)$ & $5(0)$ & $0(0)$ & $5(0)$ \\
\hline \multicolumn{6}{|l|}{ Aids } \\
\hline Não & $62.967(76,7)$ & $5.824(78,1)$ & $51.593(79,1)$ & $1.876(67,4)$ & $55.166(79,6)$ \\
\hline Sim & $8.771(10,7)$ & $568(7,6)$ & $7.367(11,3)$ & $661(23,8)$ & $7.235(10,4)$ \\
\hline Missing & $10.318(12,6)$ & $1.070(14,3)$ & $6.270(9,6)$ & $245(8,8)$ & $6.917(10,0)$ \\
\hline \multicolumn{6}{|l|}{ Diabetes mellitus } \\
\hline Não & $69.397(84,5)$ & $6.394(85,7)$ & $56.968(87,4)$ & $2.471(88,8)$ & $60.480(87,2)$ \\
\hline Sim & $5.888(7,2)$ & $105(1,4)$ & $5.116(7,8)$ & $86(3,1)$ & $5.102(7,4)$ \\
\hline Missing & $6.771(8,3)$ & $963(12,9)$ & $3.146(4,8)$ & $225(8,1)$ & $3.736(5,4)$ \\
\hline \multicolumn{6}{|l|}{ Doença mental } \\
\hline Não & $73.019(89,0)$ & $6.402(85,8)$ & $60.315(92,5)$ & $2.348(84,4)$ & $63.939(92,3)$ \\
\hline Sim & $1.967(2,4)$ & $91(1,2)$ & $1.634(2,5)$ & $187(6,7)$ & $1.532(2,2)$ \\
\hline Missing & $7.070(8,6)$ & $969(13,0)$ & $3.281(5,0)$ & $247(8,9)$ & $3.847(5,5)$ \\
\hline \multicolumn{6}{|l|}{ Alcoolismo } \\
\hline Não & $61.029(74,4)$ & $5.769(77,3)$ & $49.835(76,4)$ & $1.163(41,8)$ & $54.071(78,0)$ \\
\hline Sim & $14.626(17,8)$ & $782(10,5)$ & $12.393(19,0)$ & $1.460(52,5)$ & $11.645(16,8)$ \\
\hline Missing & $6.401(7,8)$ & $911(12,2)$ & $3.002(4,6)$ & $159(5,7)$ & $3.602(5,2)$ \\
\hline \multicolumn{6}{|l|}{ Tabagismo } \\
\hline Não & $58.410(71,2)$ & $4.906(65,7)$ & $48.665(74,6)$ & $1.401(50,4)$ & $51.959(75,0)$ \\
\hline Sim & $15.175(18,5)$ & $1.566(21,0)$ & $13.063(20,0)$ & $1.174(42,2)$ & $13.410(19,3)$ \\
\hline Missing & $8.471(10,3)$ & $990(13,3)$ & $3.502(5,4)$ & $207(7,4)$ & $3.949(5,7)$ \\
\hline \multicolumn{6}{|l|}{ Drogas } \\
\hline Não & $63.182(77,0)$ & $4.981(66,7)$ & $53.289(81,7)$ & $1.113(40,0)$ & $56.935(82,1)$ \\
\hline Sim & $9.934(12,1)$ & $1.430(19,2)$ & $8.117(12,4)$ & $1.474(53,0)$ & $8.039(11,6)$ \\
\hline Missing & $8.940(10,9)$ & $1.051(14,1)$ & $3.824(5,9)$ & $195(7,0)$ & $4.344(6,3)$ \\
\hline
\end{tabular}

na população em geral ${ }^{15}$, esse estudo mostrou que o desfecho dos casos se diferencia nessas populações.

Dados de 1993 a 2003 do Sistema Nacional de Vigilância da TB dos EUA mostraram que a PPL foi mais propensa a ter pelo menos um fator de risco para o adoecimento quando comparada a não PPL $(60,1 \%$ vs. $42,0 \%)$, além de mostrarse menos propensa a completar o tratamento, $76,8 \%$ vs. $89,4 \%{ }^{14}$. Ainda assim, a proporção de desfecho favorável na PPL dos EUA se aproximou da encontrada nesse estudo (78,6\%). Uma pes- 
Tabela 2. Características clínicas dos casos diagnosticados com tuberculose segundo o tipo de população. Brasil, 2015.

\begin{tabular}{|c|c|c|c|c|c|}
\hline Características clínicas & $\begin{array}{c}\text { População } \\
\text { total } \\
\mathbf{n}=\mathbf{8 2 . 0 5 6}(\%)\end{array}$ & $\begin{array}{c}\text { População } \\
\text { privada de } \\
\text { liberdade } \\
\mathrm{n}=7.462(\%)\end{array}$ & $\begin{array}{c}\text { População } \\
\text { não privada } \\
\text { de liberdade } \\
\mathbf{n}=65.230(\%)\end{array}$ & $\begin{array}{c}\text { População } \\
\text { em situação } \\
\text { de rua } \\
\text { n=2.782 (\%) }\end{array}$ & $\begin{array}{c}\text { População não } \\
\text { em situação } \\
\text { de rua } \\
\text { n=69.318 (\%) }\end{array}$ \\
\hline \multicolumn{6}{|l|}{ Raio $\mathrm{x}$} \\
\hline Normal & $3.745(4,6)$ & $144(1,9)$ & $3.260(5,0)$ & $73(2,6)$ & $3.301(4,8)$ \\
\hline Suspeito & $58.364(71,1)$ & $3.389(45,5)$ & $49.135(75,3)$ & $2.109(75,9)$ & $50.090(72,3)$ \\
\hline Outra patologia & $1.202(1,5)$ & $55(0,7)$ & $905(1,4)$ & $18(0,6)$ & $926(1,3)$ \\
\hline Não realizado & $15.094(18,4)$ & $3.375(45,2)$ & $9.922(15,2)$ & $491(17,6)$ & $12.646(18,2)$ \\
\hline Missing & $3.651(4,4)$ & $499(6,7)$ & $2.008(3,1)$ & $91(3,3)$ & $2.355(3,4)$ \\
\hline \multicolumn{6}{|l|}{ Baciloscopia de escarro } \\
\hline Negativa & $15.751(19,2)$ & $1.545(20,7)$ & $12.594(19,3)$ & $500(18,0)$ & $13.536(19,5)$ \\
\hline Positiva & $44.390(54,1)$ & $4.694(62,9)$ & $34.807(53,4)$ & $1.680(60,4)$ & $37.459(54,1)$ \\
\hline Não realizada & $17.648(21,5)$ & $1.057(14,2)$ & $14.014(21,5)$ & $538(19,3)$ & $14.422(20,8)$ \\
\hline Não se aplica & $4.230(5,2)$ & $166(2,2)$ & $3.814(5,8)$ & $64(2,3)$ & $3.900(5,6)$ \\
\hline Missing & $37(0)$ & $0(0)$ & $1(0)$ & $0(0)$ & $1(0)$ \\
\hline \multicolumn{6}{|l|}{ Cultura inicial } \\
\hline Negativa & $7.993(9,7)$ & $805(10,8)$ & $6.592(10,1)$ & $280(10,1)$ & $7.019(10,1)$ \\
\hline Positiva & $17.354(21,2)$ & $2.790(37,4)$ & $13.572(20,8)$ & $981(35,3)$ & $15.318(22,1)$ \\
\hline Em andamento & $2.881(3,5)$ & $432(5,8)$ & $2.174(3,3)$ & $123(4,4)$ & $2.446(3,5)$ \\
\hline Não realizada & $53.790(65,6)$ & $3.435(46,0)$ & $42.890(65,8)$ & $1.398(50,2)$ & $45.533(64,3)$ \\
\hline Missing & $38(0)$ & $0(0)$ & $2(0)$ & $0(0)$ & $2(0)$ \\
\hline \multicolumn{6}{|l|}{ Tratamento supervisionado } \\
\hline Não & $25.042(30,5)$ & $1.377(18,5)$ & $20.887(32,0)$ & $780(28,0)$ & $21.387(30,9)$ \\
\hline $\operatorname{Sim}$ & $28.045(34,2)$ & $3.180(42,6)$ & $22.328(34,2)$ & $923(33,2)$ & $24.340(35,1)$ \\
\hline Missing & $28.969(35,3)$ & $2.905(38,9)$ & $22.015(33,8)$ & $1.079(38,8)$ & $23.591(34,0)$ \\
\hline \multicolumn{6}{|l|}{ Desfecho do caso } \\
\hline Cura & $53.381(65)$ & $5.068(67,9)$ & $42.952(65,9)$ & $965(34,7)$ & $46.658(67,4)$ \\
\hline Perda de seguimento & $9.106(11,1)$ & $567(7,6)$ & $7.515(11,5)$ & $938(33,7)$ & $7.112(10,3)$ \\
\hline Óbito por TB & $2.981(3,6)$ & $72(1,0)$ & $2.336(3,6)$ & $164(5,9)$ & $2.235(3,2)$ \\
\hline Óbito por outras causas & $3.588(4,4)$ & $117(1,6)$ & $3.029(4,6)$ & $159(5,7)$ & $2.971(4,3)$ \\
\hline Transferência & $4.818(5,9)$ & $478(6,4)$ & $3.548(5,4)$ & $236(8,5)$ & $3.749(5,4)$ \\
\hline TB-MDR & $893(1,1)$ & $99(1,3)$ & $727(1,1)$ & $33(1,2)$ & $786(1,1)$ \\
\hline Mudança de esquema & $406(0,5)$ & $14(0,2)$ & $371(0,6)$ & $12(0,4)$ & $374(0,5)$ \\
\hline Falência & $77(0,1)$ & $0(0)$ & $66(0,1)$ & $3(0,1)$ & $62(0,1)$ \\
\hline $\begin{array}{l}\text { Perda de seguimento } \\
\text { primário }\end{array}$ & $591(0,7)$ & $31(0,4)$ & $507(0,8)$ & $110(4,0)$ & $427(0,6)$ \\
\hline Missing & $6.215(7,6)$ & $1.016(13,6)$ & $4.179(6,4)$ & $162(5,8)$ & $4.944(7,1)$ \\
\hline
\end{tabular}

quisa realizada em El Salvador de 2009 a 2013, apontou indicadores de sucesso no tratamento da TB em prisões superiores a $95 \%{ }^{16}$.

Não foram encontradas outras pesquisas no Brasil que mostrassem a associação da PPL com o desfecho favorável nos casos como demostrado (protetor para insucesso) (RC 0,68, IC95\% 0,63-0,73), porém considera-se que esse achado pode estar associado ao efeito benéfico da realização do tratamento diretamente observado.
Uma pesquisa realizada no Brasil demostrou que a realização do TDO diminui em $25 \%$ os desfechos desfavoráveis no tratamento da $\mathrm{TB}^{17}$. Neste estudo, o TDO mostrou-se associado ao sucesso no desfecho dos casos tanto na população privada de liberdade quanto na população em situação de rua.

Por outro lado, são muitos os fatores dificultadores para o tratamento contínuo da tuberculose na população em situação de rua, tais como: 


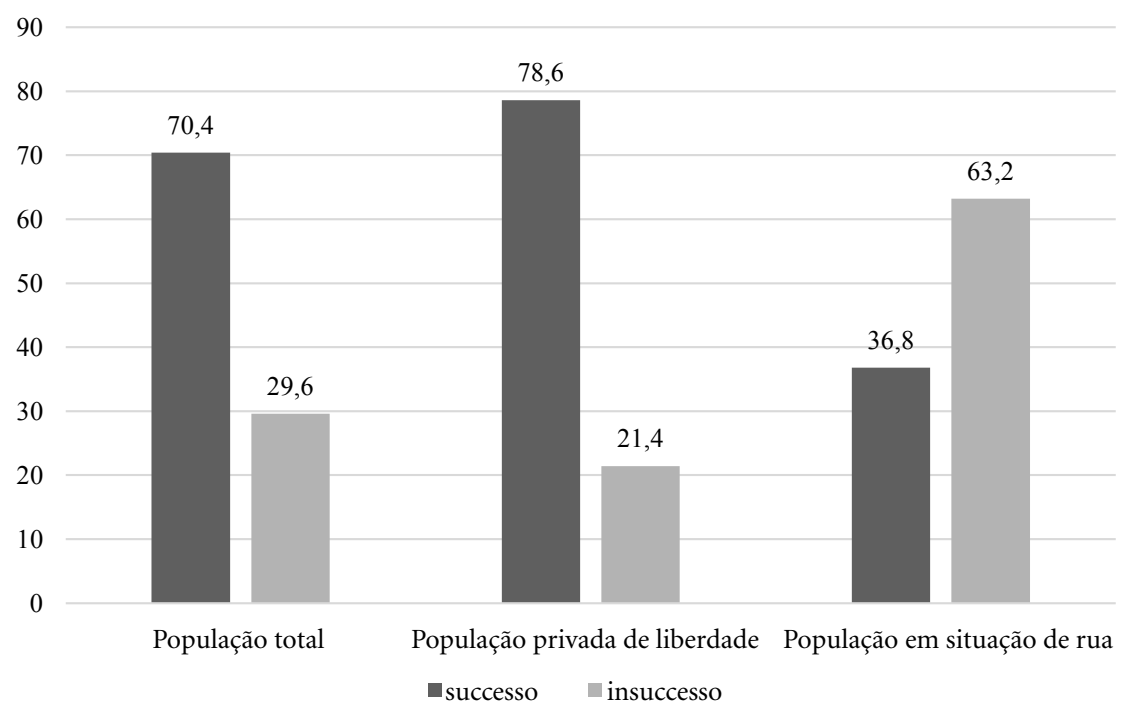

Gráfico 1. Desfecho dos casos de tuberculose categorizado em sucesso e insucesso (\%) segundo o tipo de população. Brasil, 2015.

Fonte: Elaborado pelos autores.

alimentação insuficiente, agravos de saúde associados, uso de álcool e outras drogas, baixa autoestima, dificuldade de percepção dos sintomas, e a própria dinâmica do ambiente da rua que dificulta que as medicações sejam tomadas com regularidade e facilita o roubo/perda dos medicamentos ${ }^{18}$.

Este estudo mostrou para a população em situação de rua uma proporção de $63,2 \%$ de insucesso no desfecho dos casos e uma razão de chances ajustada de 2,38 (IC95\% 2,17-2,61) para desfechos desfavoráveis (insucesso). Resultados semelhantes foram observados em outros estudos: em São Paulo' ${ }^{9}$ demostrou-se 57,3\% de insucesso e RC de 4,96 (IC95\% 4,27-5,76); em Londres ${ }^{19}$ a população em situação de rua mostrou-se associada a multidroga resistência (RC 2,1), falta de adesão (RC 2,5) e a perda de seguimento (RC 3,8 ); e na Nicarágua ${ }^{20}$, onde a população em situação de rua mostrou-se associada a perda de seguimento do tratamento (RC 3,08, IC95\% $1,57-6,49)$.

Uma coorte realizada nos EUA no período de 1994 a 2010 mostrou que a população em situação de rua apresentou duas vezes mais chances de não completar o tratamento ${ }^{21}$. Os casos eram em sua maioria do sexo masculino, adultos jovens e com alta prevalência de uso de álcool, drogas e positividade para o $\mathrm{HIV}^{21}$, assim como observado nesse estudo.

É importante enfatizar que questões relacionadas a dificuldade de acesso aos equipamentos de saúde como falta de flexibilidade e regras rígidas para os dias e horários de atendimento, agravam ainda mais essa questão ${ }^{18}$.

Esse estudo apresenta limitações. A incorporação da variável que aponta a situação de rua na ficha de notificação do Sinan (situações especiais) foi realizada no ano de 2014, e dessa forma o quantitativo da população em situação de rua em 2015 pode estar subestimado, haja vista o período natural de transição dos profissionais notificadores para a adaptação a nova variável. Além disso, tanto a população em situação de rua quanto a população privada de liberdade são grupos estigmatizados socialmente, o que pode dificultar que o indivíduo diagnosticado se declare pertencente a esses grupos ou se permita ser identificado no momento da notificação.

Embora o Programa Nacional de Tuberculose lance mão de ferramentas para o controle da qualidade do banco de dados do Sinan TB ainda é possível observar um quantitativo elevado de dados incompletos em algumas variáveis especi- 
Tabela 3. Associação entre estar privado de liberdade ou em situação de rua e o insucesso no desfecho dos casos de tuberculose ajustado pelas variáveis no modelo de regressão logística. Brasil, 2015.

\begin{tabular}{|c|c|c|c|c|}
\hline Características & $\begin{array}{c}\text { População } \\
\text { privada de } \\
\text { liberdade } \\
\text { RCa } \\
\end{array}$ & IC95\% & $\begin{array}{c}\text { População } \\
\text { em situação } \\
\text { de rua } \\
\text { RCa }\end{array}$ & IC95\% \\
\hline \multicolumn{5}{|l|}{ Populações vulneráveis } \\
\hline Não & referência & & referência & \\
\hline Sim & 0,68 & $0,63-0,73$ & 2,38 & $2,17-2,61$ \\
\hline \multicolumn{5}{|l|}{ Ajustado por } \\
\hline \multicolumn{5}{|l|}{ Sexo } \\
\hline Masculino & referência & & referência & \\
\hline Feminino & 0,84 & $0,80-0,87$ & 0,87 & $0,83-0,90$ \\
\hline \multicolumn{5}{|l|}{ Faixa etária } \\
\hline 15 a 39 anos & referência & & referência & \\
\hline 40 a 64 anos & 0,98 & $0,94-1,02$ & 1,01 & $0,97-1,05$ \\
\hline 65 ou mais anos & 1,49 & $1,39-1,60$ & 1,57 & $1,46-1,68$ \\
\hline \multicolumn{5}{|l|}{ Raça/Cor } \\
\hline Branca & referência & & referência & \\
\hline Preta & 1,22 & $1,15-1,29$ & 1,20 & $1,14-1,28$ \\
\hline Amarela & 1,10 & $0,89-1,35$ & 1,09 & $0,88-1,33$ \\
\hline Parda & 1,21 & $1,15-1,27$ & 1,20 & $1,15-1,26$ \\
\hline Indígena & 1,17 & $0,96-1,41$ & 1,21 & $0,94-1,45$ \\
\hline \multicolumn{5}{|l|}{ Escolaridade } \\
\hline Analfabeto & referência & & referência & \\
\hline 1 a 4 anos & 0,80 & $0,73-0,89$ & 0,81 & $0,73-0,89$ \\
\hline 5 a 8 anos & 0,77 & $0,69-0,85$ & 0,78 & $0,70-0,86$ \\
\hline$>8$ anos & 0,57 & $0,51-0,63$ & 0,59 & $0,53-0,65$ \\
\hline Não se aplica & 0,75 & $0,60-0,94$ & 0,76 & $0,60-0,95$ \\
\hline \multicolumn{5}{|l|}{ Região } \\
\hline Norte & referência & & referência & \\
\hline Nordeste & 1,19 & $1,11-1,28$ & 1,18 & $1,10-1,26$ \\
\hline Sudeste & 1,12 & $1,04-1,20$ & 1,09 & $1,01-1,17$ \\
\hline Sul & 1,40 & $1,28-1,53$ & 1,37 & $1,23-1,48$ \\
\hline Centro-Oeste & 1,30 & $1,17-1,44$ & 1,26 & $1,13-1,40$ \\
\hline \multicolumn{5}{|l|}{ Entrada } \\
\hline Caso novo & referência & & referência & \\
\hline Recidiva & 1,24 & $1,15-1,32$ & 1,21 & $1,13-1,30$ \\
\hline Reingresso após perda de seguimento & 3,32 & $3,13-3,51$ & 3,16 & $2,98-3,35$ \\
\hline Não sabe & 2,68 & $1,97-3,63$ & 2,54 & $1,86-3,45$ \\
\hline Transferência & 2,11 & $1,91-2,32$ & 2,08 & $1,88-2,29$ \\
\hline Pós-óbito & 1313,87 & $\begin{array}{l}7,36 \mathrm{e}-09- \\
2,34 \mathrm{e}+14\end{array}$ & 1337,84 & $\begin{array}{l}7,36 \mathrm{e}-09 \\
2,34 \mathrm{e}+14\end{array}$ \\
\hline
\end{tabular}

ficas, por isso optou-se por utilizar-se a técnica de imputação múltipla dos dados nesse estudo. Foram realizadas comparações entre as análises com os dados imputados e a análise com os dados completos, que se apresentaram semelhantes quanto aos valores encontrados. Vale lembrar que o Sinan é um sistema de informação nacio- nal bem estruturado que apresenta como principais vantagens para seu uso, o baixo custo na coleta dos dados e a ampla cobertura ${ }^{6}$.

A partir da necessidade de implementação de uma política pública de inclusão social que considere a promoção dos direitos humanos das pessoas privadas de liberdade e suas demandas, foi 
Tabela 3. Associação entre estar privado de liberdade ou em situação de rua e o insucesso no desfecho dos casos de tuberculose ajustado pelas variáveis no modelo de regressão logística. Brasil, 2015.

\begin{tabular}{|c|c|c|c|c|}
\hline Características & $\begin{array}{c}\text { População } \\
\text { privada de } \\
\text { liberdade } \\
\text { RCa } \\
\end{array}$ & IC95\% & $\begin{array}{c}\text { População } \\
\text { em situação } \\
\text { de rua } \\
\text { RCa }\end{array}$ & IC95\% \\
\hline \multicolumn{5}{|l|}{ Forma } \\
\hline Pulmonar & referência & & referência & \\
\hline Extrapulmonar & 0,83 & $0,77-0,88$ & 0,85 & $0,79-0,91$ \\
\hline Pulmonar+Extrapulmonar & 1,19 & $1,07-1,31$ & 1,21 & $1,09-1,33$ \\
\hline \multicolumn{5}{|l|}{ Aids } \\
\hline Não & referência & & referência & \\
\hline $\operatorname{Sim}$ & 2,93 & $2,77-3,10$ & 2,92 & $2,76-3,08$ \\
\hline \multicolumn{5}{|l|}{ Diabetes mellitus } \\
\hline Não & referência & & referência & \\
\hline Sim & 1,00 & $0,93-1,06$ & 1,02 & $0,95-1,08$ \\
\hline \multicolumn{5}{|l|}{ Doença mental } \\
\hline Não & referência & & referência & \\
\hline Sim & 1,16 & $1,04-1,29$ & 1,13 & $1,01-1,25$ \\
\hline \multicolumn{5}{|l|}{ Alcoolismo } \\
\hline Não & referência & & referência & \\
\hline Sim & 1,39 & $1,32-1,45$ & 1,38 & $1,31-1,44$ \\
\hline \multicolumn{5}{|l|}{ Tabagismo } \\
\hline Não & referência & & referência & \\
\hline Sim & 1,08 & $1,03-1,14$ & 1,08 & $1,02-1,13$ \\
\hline \multicolumn{5}{|l|}{ Drogas } \\
\hline Não & referência & & referência & \\
\hline Sim & 1,68 & $1,59-1,79$ & 1,54 & $1,44-1,63$ \\
\hline \multicolumn{5}{|l|}{ Raio $\mathrm{x}$} \\
\hline Normal & referência & & referência & \\
\hline Suspeito & 1,08 & $0,99-1,19$ & 1,10 & $1,00-1,20$ \\
\hline Outra patologia & 1,51 & $1,29-1,77$ & 1,51 & $1,29-1,77$ \\
\hline Não realizado & 1,12 & $1,01-1,24$ & 1,07 & $0,97-1,18$ \\
\hline \multicolumn{5}{|l|}{ Baciloscopia de escarro } \\
\hline Negativa & referência & & referência & \\
\hline Positiva & 0,93 & $0,88-0,97$ & 0,94 & $0,89-0,99$ \\
\hline Não realizada & 1,17 & $1,11-1,24$ & 1,19 & $1,12-1,25$ \\
\hline Não se aplica & 1,00 & $0,91-1,11$ & 1,03 & $0,93-1,13$ \\
\hline \multicolumn{5}{|l|}{ Cultura inicial } \\
\hline Negativa & referência & & referência & \\
\hline Positiva & 1,52 & $1,41-1,63$ & 1,48 & $1,38-1,59$ \\
\hline Em andamento & 3,90 & $3,48-4,37$ & 3,80 & $3,39-4,25$ \\
\hline Não realizada & 1,64 & $1,53-1,75$ & 1,65 & $1,55-1,76$ \\
\hline \multicolumn{5}{|l|}{ Tratamento supervisionado } \\
\hline Não & referência & & referência & \\
\hline $\operatorname{Sim}$ & 0,47 & $0,45-0,48$ & 0,46 & $0,44-0,48$ \\
\hline
\end{tabular}

RCa - Razão de chances ajustada.

Fonte: Elaborado pelos autores.

instituído no ano de 2003 pelos Ministérios da Saúde e da Justiça, o Plano Nacional de Saúde no Sistema Penitenciário (PNSSP), fomentando a reorientação do modelo assistencial para essa po- 
blicada na Portaria Interministerial no 1 , de 2 de janeiro de 2014, que possui como objetivo principal garantir o acesso das pessoas privadas de liberdade ao cuidado integral no Sistema Único de Saúde (SUS). A PNAISP prevê que os serviços de saúde no sistema prisional sejam pontos de atenção da Rede de Atenção à Saúde (RAS) do SUS, qualificando também a Atenção Básica no âmbito prisional como porta de entrada do sistema e ordenadora das ações e serviços de saúde pela rede $^{22,23}$. Essas políticas contribuíram de forma significativa para a melhoria da assistência à saúde no sistema prisional, bem como para o diagnóstico, tratamento e controle da tuberculose.

É essencial ressaltar que os principais fatores relacionados ao contexto da tuberculose nas populações privada de liberdade e em situação de rua estão associados principalmente aos aspectos sociais e institucionais, provenientes da desigualdade social e barreiras de acesso aos serviços de saúde. Nessa lógica, a implantação de ações que abarquem somente questões biológicas tornamse insuficientes para o controle da doença.

Programas de transferência de renda têm contribuído de maneira efetiva para a diminui- ção da incidência da $\mathrm{TB}^{24}$ e para cura da doença $^{25}$. Também já são registrados os benefícios da priorização da tomada da medicação da TB de forma observada, sendo assim sugerida a expansão dessa estratégia, principalmente para a população em situação de rua. Em 2011, foi instituída pela Política Nacional de Atenção Básica (PNAB), o Consultório na Rua com o objetivo de ampliar o acesso da população em situação de rua aos serviços de saúde, ofertando, de maneira mais oportuna, atenção integral à saúde para esse grupo. O Consultório na Rua conta com equipes multiprofissionais que desenvolvem ações de forma itinerante e em parceria com as equipes das Unidades Básicas de Saúde do território ${ }^{18}$, atuando como um importante aliado para a realização do TDO na população em situação de rua.

Diante da complexidade do cenário exposto, faz-se necessário a implantação e ampliação de políticas públicas de saúde que considerem as especificidades dessas populações e que principalmente seja articulada com órgãos sociais e de segurança a fim de que de fato impacte nos indicadores da tuberculose.

\section{Colaboradores}

LR Macedo trabalhou na concepção, redação do artigo e revisão crítica. ELN Macedo e CJ Struchiner trabalharam na concepção, revisão crítica e aprovação da versão a ser publicada. 


\section{Referências}

1. World Health Organization (WHO). Global Tuberculosis Report 2016. Genebra: WHO; 2016.

2. San Pedro A, Oliveira RM. Tuberculosis and socioeconomic indicators: systematic review of the literature Rev Panam Salud Publica 2013; 33(4):294-301.

3. Brasil. Ministério da Saúde (MS). Populações Vulneráveis - Tuberculose [Internet]. [acessado 2017 jul 25]. Disponível em: http://portalsaude.saude.gov.br/ index.php/o-ministerio/principal/leia-mais-o-ministerio/743-secretaria-svs/vigilancia-de-a-a-z/tuberculose/12-tuberculose/11941-viajantes-tuberculose.

4. Maciel EL, Reis-Santos B. Determinants of tuberculosis in Brazil: from conceptual framework to practical application. Rev Panam Salud Publica 2015; 38(1):2834.

5. Maciel ELN. Estratégias da agenda pós-2015 para o controle da tuberculose no Brasil: desafios e oportunidades. Epidemiol Serv Saude 2016; 25(2):423-436.

6. Malhão TA, Oliveira GP, Codennoti S, Moherdaui F Avaliação da completitude do Sistema de Informação de Agravos de Notificação da Tuberculose, Brasil, 2001-2006. Epidemiol Serv Saude 2010; 19(3):245-256.

7. Harrell FE. Regression Modeling Strategies [Internet]. Cham: Springer International Publishing; 2015.

8. Macedo LR, Reis-Santos B, Riley LW, Maciel EL. Treatment outcomes of tuberculosis patients in Brazilian prisons: a polytomous regression analysis. Int $J$ Tuberc Lung Dis 2013; 17(11):1427-1434.

9. Ranzani OT, Carvalho CRR, Waldman EA, Rodrigues LC. The impact of being homeless on the unsuccessful outcome of treatment of pulmonary TB in São Paulo State, Brazil. BMC Med 2016; 14:41.

10. Hargreaves JR, Boccia D, Evans CA, Adato M, Petticrew M, Porter JDH. The Social Determinants of Tuberculosis: From Evidence to Action. Am J Public Health 2011; 101(4):654-662.

11. Feske ML, Teeter LD, Musser JM, Graviss EA. Counting the Homeless: A Previously Incalculable Tuberculosis Risk and Its Social Determinants. Am J Public Health 2013; 103(5):839-848.

12. Halpern SC, Scherer JN, Roglio V, Faller S, Sordi A, Ornell F, Dalbosco C, Pechansky F, Kessler F, von Diemen L. Clinical and social vulnerabilities in crack users according to housing status: a multicenter study in six Brazilian state capitals. Cad Saude Publica 2017; 33(6):e00037517.

13. Grangeiro A, Holcman MM, Onaga ET, Alencar HDR, Placco ALN, Teixeira PR. Prevalence and vulnerability of homeless people to HIV infection in São Paulo, Brazil. Rev Saude Publica 2012; 46(4):674-684

14. MacNeil JR, Lobato MN, Moore M. An unanswered health disparity: tuberculosis among correctional inmates, 1993 through 2003. Am J Public Health 2005; 95(10):1800-1805

15. Brasil. Ministério da Saúde (MS). Secretaria de Vigilância em Saúde. Departamento de Vigilância das Doenças Transmissíveis. Manual de recomendações para o controle da tuberculose no Brasil. Brasília: MS; 2019.
16. Ayala G, Garay J, Aragon M, Decroo T, Zachariah R Trends in tuberculosis notification and treatment outcomes in prisons: a country-wide assessment in El Salvador from 2009-2014. Rev Panam Salud Publica 2016; 39(1):38-43.

17. Reis-Santos B, Pellacani-Posses I, Macedo LR, Golub JE, Riley LW, Maciel EL. Directly observed therapy of tuberculosis in Brazil: associated determinants and impact on treatment outcome. Int J Tuberc Lung Dis 2015; 19(10):1188-1193.

18. Brasil. Ministério da Saúde (MS). Secretaria de Atenção à Saúde. Departamento de Atenção Básica. Manual sobre o cuidado à saúde junto a população em situação de rua. Brasília: MS; 2012.

19. Story A, Murad S, Roberts W, Verheyen M, Hayward AC. Tuberculosis in London: the importance of homelessness, problem drug use and prison. Thorax 2007; 62(8):667.

20. Soza Pineda NI, Pereira SM, Barreto ML. Dropout from tuberculosis treatment in Nicaragua: the results of a comparative study. Rev Panam Salud Publica 2005; 17(4):271-278

21. Bamrah S, Woodruff RSY, Powell K, Ghosh S, Kammerer JS, Haddad MB. Tuberculosis among the homeless, United States, 1994-2010. Int J Tuberc Lung Dis 2013; 17(11):1414-1419.

22. Brasil. Ministério da Saúde (MS). Departamento de Ações Programáticas Estratégicas. Área Técnica de Saúde no Sistema Penitenciário. Plano Nacional de Saúde no Sistema Penitenciário (PNSSP). Brasília: MS 2004.

23. Brasil. Ministério da Saúde (MS). Secretaria de Atenção à Saúde. Departamento de Ações Programáticas Estratégicas. Coordenação de Saúde no Sistema Prisional. Legislação em Saúde no Sistema Prisional. Brasília: MS; 2014

24. Nery JS, Rodrigues LC, Rasella D, Aquino R, Barreira D, Torrens AW, Boccia D, Penna GO, Penna MLF Barreto ML, Pereira SM. Effect of Brazil's conditional cash transfer programme on tuberculosis incidence. Int J Tuberc Lung Dis 2017; 21(7):790-796.

25. Torrens AW, Rasella D, Boccia D, Maciel ELN, Nery JS, Olson ZD, Barreira DCN, Sanchez MN. Effectiveness of a conditional cash transfer programme on TB cure rate: a retrospective cohort study in Brazil. Trans $R$ Soc Trop Med Hyg 2016; 110(3):199-206.

Artigo apresentado em 12/05/2020

Aprovado em 13/08/2020

Versão final apresentada em 15/08/2020

Editores-chefes: Romeu Gomes, Antônio Augusto Moura da Silva 
